\title{
Rare or forbidden decays at Belle
}

\author{
Oksana Brovchenko \\ IEKP, Karlsruhe Insitute of Technology \\ E-mail: oksana.brovchenko@kit.edu
}

\begin{abstract}
We report three searches for rare or forbidden decays at Belle. Search for rare decays offers an opportunity to search for New Physics beyond the Standard Model.

The results are obtained from $B \bar{B}$ pair events collected at the $\Upsilon(4 S)$ resonance with the Belle detector at the KEKB $e^{+} e^{-}$collider. A search for $B^{0}$ decays into invisible final states was performed. No significant signal was observed and an upper limit of $1.3 \times 10^{-4}$ at $90 \% \mathrm{CL}$ was obtained. In the second analysis a search for $B \rightarrow h^{(*)} v \bar{v}$ decays, where $h^{(*)}$ stands for $K^{+}, K_{S}^{0}$, $K^{*+}, K^{* 0}, \pi^{+}, \pi^{0}, \rho^{+}, \rho^{0}$ and $\phi$, was performed. The sensitivity was improved compared to the previous Belle analysis. No significant signal was observed, four of the calculated upper limits provide worlds currently most restructive limits. We also report a search for heavy neutral lepton in $B$ meson decays. No signal is observed and upper limits are set on mixing of heavy neutral leptons with left-handed neutrinos.
\end{abstract}

36th International Conference on High Energy Physics,

July 4-11, 2012

Melbourne, Australia 


\section{1. $B^{0}$ decays to invisible final states}

The decay $B^{0} \rightarrow v \bar{v}$ is highly helicity supressed in the Standard Model (SM). The Feynman graphs for this decays are shown in fig. 1a. The expected branching fraction is very small: $\mathscr{B} \sim \mathscr{O}\left(10^{-20}\right)[1]$. The experimental signature of such decay at would be missing energy and momentum, which correspond exactly to the presence of a $B^{0}$ meson. There are also processes including new particles beyond the SM, which can have the same experimental signature. One example of such a decay includes a supersymmetric neutralino: $B^{0} \rightarrow \bar{v} \chi_{1}^{0}$. The Feynman graphs for this decay are shown in fig. $1 \mathrm{~b}$ and the expected branching fraction is calculated to be $\mathscr{B} \sim \mathscr{O}\left(10^{-6}-10^{-7}\right)$ [2], and is thus several order of magnitude larger than the SM expectations. An observation of a $B^{0}$ going to invisible final states could lead to a discovery of New Physics. The data sample of

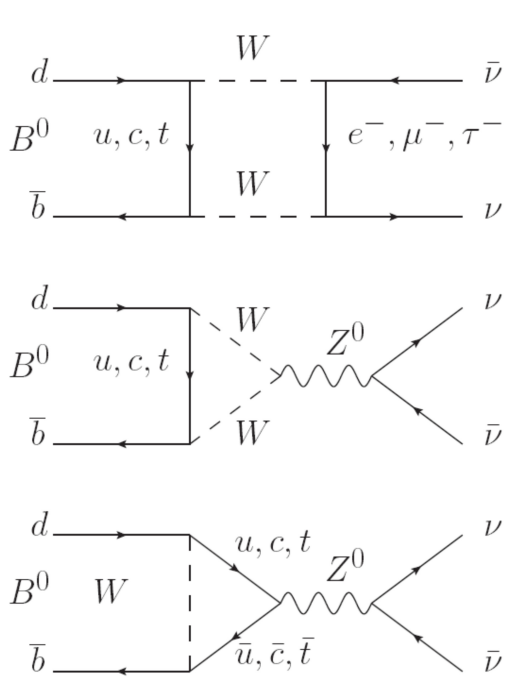

(a) Standard Model
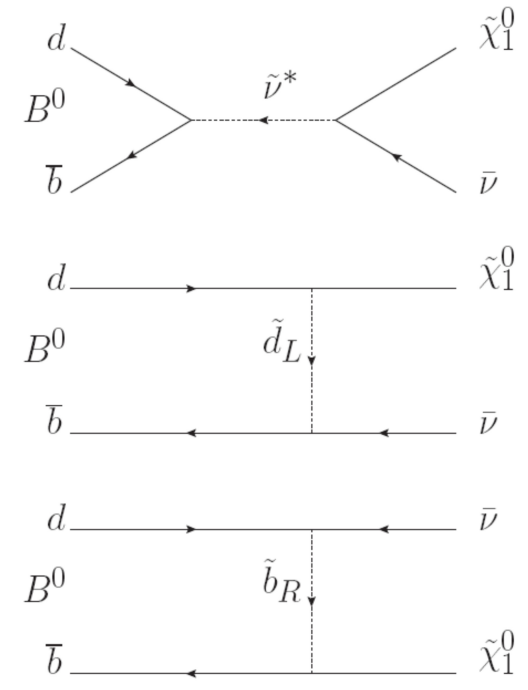

(b) New Physics models

Figure 1: Feynman diagramms for the $B \rightarrow v \bar{v}$ in the Standard Model (left) and in the R-parity violating models (right).

$605 \mathrm{fb}^{-1}$ collected at the $\Upsilon(4 S)$ resonance at Belle was used for this analysis, this corresponds to $657 \times 10^{6} B \bar{B}$ pairs. One $B$ meson, $B_{t a g}$, was fully reconstructed in hadronic modes. After the full reconstruction $9.5 \times 10^{5}$ neutral $B_{\text {tag }}$ candidates were obtained. Further candidate selection required no additional charged tracks, $\pi^{0}$ or $K_{L}^{0}$ candidates left in the event. The continuum background $\left(e^{+} e^{-} \rightarrow q \bar{q}\right)$ was suppressed using the following two variables. The angle between the $B_{\text {tag }}$ thrust axis and the beam axis in the $\mathrm{CM}$ frame, $\cos \theta_{T}$, was used to cut away a large amount of continuum background. The angle between the $B_{\text {tag }}$ flight direction and the beam axis in the CM frame, $\cos \theta_{B}$, was used to extract the signal yield. The most powerful variable to separate signal from background is the sum of the remaining energie clusters in the electromagnetic calorimeter that are not associated with $B_{t a g}$ tracks or neutrals, $E_{E C L}$. The signal yield is extracted from an extended unbinned maximum likelihood fit to $E_{E C L}$ and $\cos \theta_{B}$. A validation of the $E_{E C L}$ simulation was performed using doubly tagged samples hadronic $B_{t a g}+B^{0} \rightarrow D^{(*)-} l^{+} v$, a good agreement 
between data and Monte Carlo was observed.

The final fit results on data are shown in fig. 2. The observed number of signal events was $N_{\text {sig }}=8.9_{-5.5}^{+6.3}$, so no significant signal was observed. The evaluated upper limit at $90 \% \mathrm{CL}$ is $\mathscr{B}(B \rightarrow$ invisible $)<1.3 \times 10^{-4}$.
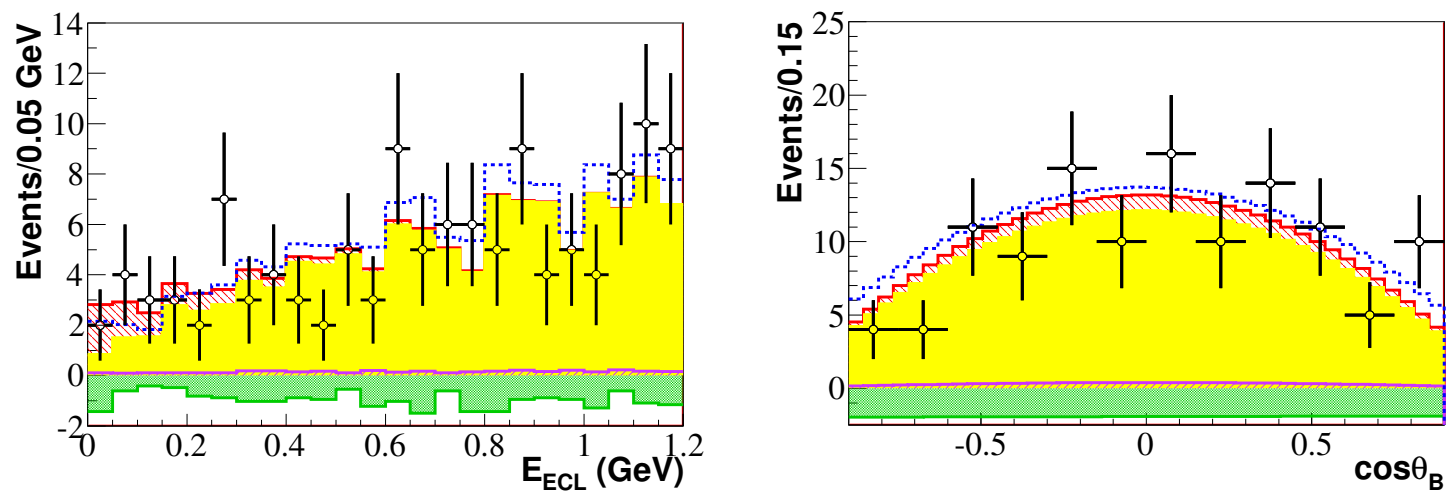

Figure 2: The results of the fit to $E_{E C L}$ and $\cos \theta_{B}$. The red line shows the signal PDF and the yellow line the total background PDF. The background components are marked as follows: blue shows $B \bar{B}$ background, pink the rare background and green the continuum background.

\section{Decays $B \rightarrow h^{(*)} v \bar{v}$}

The flavour changing neutral current transitions like $b \rightarrow s$ or $b \rightarrow d$ are forbidden at tree level in the SM. Higher order diagrams are possible, as shown in fig. 3, but they are highly suppressed. The decay $B \rightarrow h^{(*)} v \bar{v}$, which proceed via such FCNC processes have the theoretical advantage of only one charged hadron in the final state and no charged leptons. The absence of long-distance interactions allows very precise and reliable theoretical predictions, the expected branching fractions is $\mathscr{B} \sim \mathscr{O}\left(10^{-6}\right)$ at most [3]. Several models beyond the SM, for example supersymmetry, nonstandard $Z$-coupling or a forth generation, could enhance the branching fractions of these decays.

Compared to the previous analysis of these decays withing the Belle collaboration, several im-
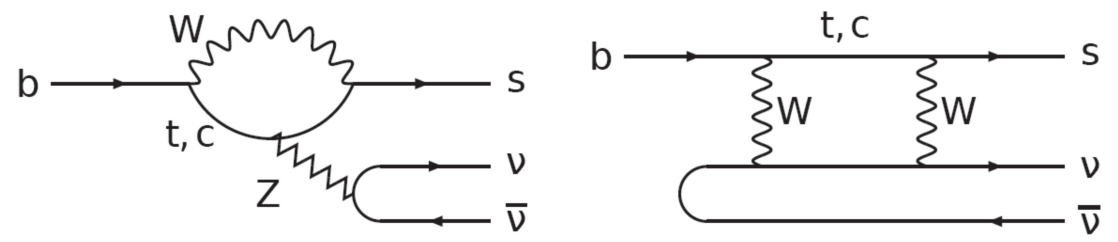

Figure 3: Feynman diagrams for the $b \rightarrow s$ transitions in the Standard Model.

provements were made. The used data sample consists of $711 \mathrm{fb}^{-1}$ collected at the $\Upsilon(4 S)$ resonance (771 $\times 10^{6} B \bar{B}$ pairs). One $B$ meson, the $B_{\text {tag }}$, was fully reconstructed in hadronic modes using the new probabilistic full reconstruction [4]. Exclusive reconstruction of 1104 decay channels and a 
multivariate approach using the neural net package NeuroBayes instead of cuts lead to efficiency improvement of factor $\sim 2$ for the same purity. After the $B_{\text {tag }}$ reconstruction, the search for one light meson, $K^{+}, K^{*+}, K_{s}^{0}, K^{* 0}, \pi^{+}, \pi^{0}, \rho^{+}, \rho^{0}$ or $\phi$ was performed. No additional charged tracks or $\pi^{0}$ candidates were allowed in the event. The continuum background $\left(e^{+} e^{-} \rightarrow q \bar{q}\right)$ was supressed using the angle between the $B_{\text {tag }}$ thrust axis and the beam axis in the CM frame, $\cos \theta_{T}$. The most powerful variable to distinguish between signal and background was the sum of the remaining energy clusters in the electromagnetic calorimeter that are not associated with $B_{t a g}$ tracks or neutrals. The signal yield was extracted from an extended binned maximum likelihood fit to $E_{E C L}$.

Using simulated Monte Carlo experiments the expected limits in case of no signal observation were evaluated and compared with the expected limits of the previous Belle analysis. The results are shown in the first columns of table 1 . It can be observed that the sensitivity of the analysis could be significantly improved. The final fits of the data distributions in $E_{E C L}$ are shown in fig. 4. No significant signal was observed, although the significance for the $K^{+}$and $\pi^{+}$channels was over $2 \sigma$. The evaluated limits are shown in table 1 , most of the limits could be improved compared to the previous Belle analysis and four limits are most stringent to date.

\begin{tabular}{|l|l|l||l||l|l|}
\hline Channel & $\begin{array}{l}\text { Expected } \\
\text { branching ratio } \\
\text { limit at 90\% } \\
\text { CL }\left(711 \mathrm{fb}^{-1}\right)\end{array}$ & $\begin{array}{l}\text { Expected } \\
\text { branching ratio } \\
\text { limit at 90\% } \\
\text { CL previous } \\
\text { Belle analysis } \\
\left(492 \mathrm{fb}^{-1}\right)[5]\end{array}$ & $\begin{array}{l}\text { Observed } \\
\text { branching ratio } \\
\text { limit at 90\% } \\
\text { CL }\end{array}$ & $\begin{array}{l}\text { Observed } \\
\text { branching ratio } \\
\text { limit at 90\% } \\
\text { CL previous } \\
\text { Belle analysis } \\
\text { [5] }\end{array}$ & $\begin{array}{l}\text { PDG limit at } \\
90 \% \text { CL }\end{array}$ \\
\hline$B^{+} \rightarrow K^{+} v \bar{v}$ & $2.2 \times 10^{-5}$ & $10 \times 10^{-5}$ & $5.5 \times 10^{-5}$ & $1.4 \times 10^{-5}$ & $1.3 \times 10^{-5}$ \\
\hline$B^{+} \rightarrow K^{*+} v \bar{v}$ & $5.3 \times 10^{-5}$ & $22 \times 10^{-5}$ & $4.0 \times 10^{-5}$ & $14 \times 10^{-5}$ & $8 \times 10^{-5}$ \\
\hline$B^{+} \rightarrow \pi^{+} v \bar{v}$ & $3.9 \times 10^{-5}$ & $10 \times 10^{-5}$ & $9.8 \times 10^{-5}$ & $17 \times 10^{-5}$ & $10 \times 10^{-5}$ \\
\hline$B^{+} \rightarrow \rho^{+} v \bar{v}$ & $9.8 \times 10^{-5}$ & $19 \times 10^{-5}$ & $21.4 \times 10^{-5}$ & $44 \times 10^{-5}$ & $15 \times 10^{-5}$ \\
\hline$B^{0} \rightarrow K_{s}^{0} v \bar{v}$ & $7.3 \times 10^{-5}$ & $16 \times 10^{-5}$ & $9.7 \times 10^{-5}$ & $16 \times 10^{-5}$ & $5.6 \times 10^{-5}$ \\
\hline$B^{0} \rightarrow K^{* 0} v \bar{v}$ & $5.0 \times 10^{-5}$ & $20 \times 10^{-5}$ & $5.4 \times 10^{-5}$ & $34 \times 10^{-5}$ & $1.2 \times 10^{-5}$ \\
\hline$B^{0} \rightarrow \pi^{0} v \bar{v}$ & $3.6 \times 10^{-5}$ & $10 \times 10^{-5}$ & $6.9 \times 10^{-5}$ & $22 \times 22^{-5}$ & $22 \times 10^{-5}$ \\
\hline$B^{0} \rightarrow \rho^{0} v \bar{v}$ & $16.5 \times 10^{-5}$ & $16 \times 10^{-5}$ & $20.8 \times 10^{-5}$ & $44 \times 10^{-5}$ & $44 \times 10^{-5}$ \\
\hline$B^{0} \rightarrow \phi v \bar{v}$ & $9.1 \times 10^{-5}$ & $13 \times 10^{-5}$ & $12.7 \times 10^{-5}$ & $5.8 \times 10^{-5}$ & $5.8 \times 10^{-5}$ \\
\hline
\end{tabular}

Table 1: Expected and observed limits of the presented analysis, compared with the previous Belle analysis and current PDG values.

\section{Search for heavy neutral lepton}

In the SM masses are generated via the coupling of the Higgs boson to left and right components of the particle. There are no right-handed neutrinos in the SM, which implies that neutrinos should be massless. Neutrino oszillations show however, that neutrinos do have a mass. An existence of sterile right handed neutrinos might solve this issue. Heavy neutral leptons also appear in 

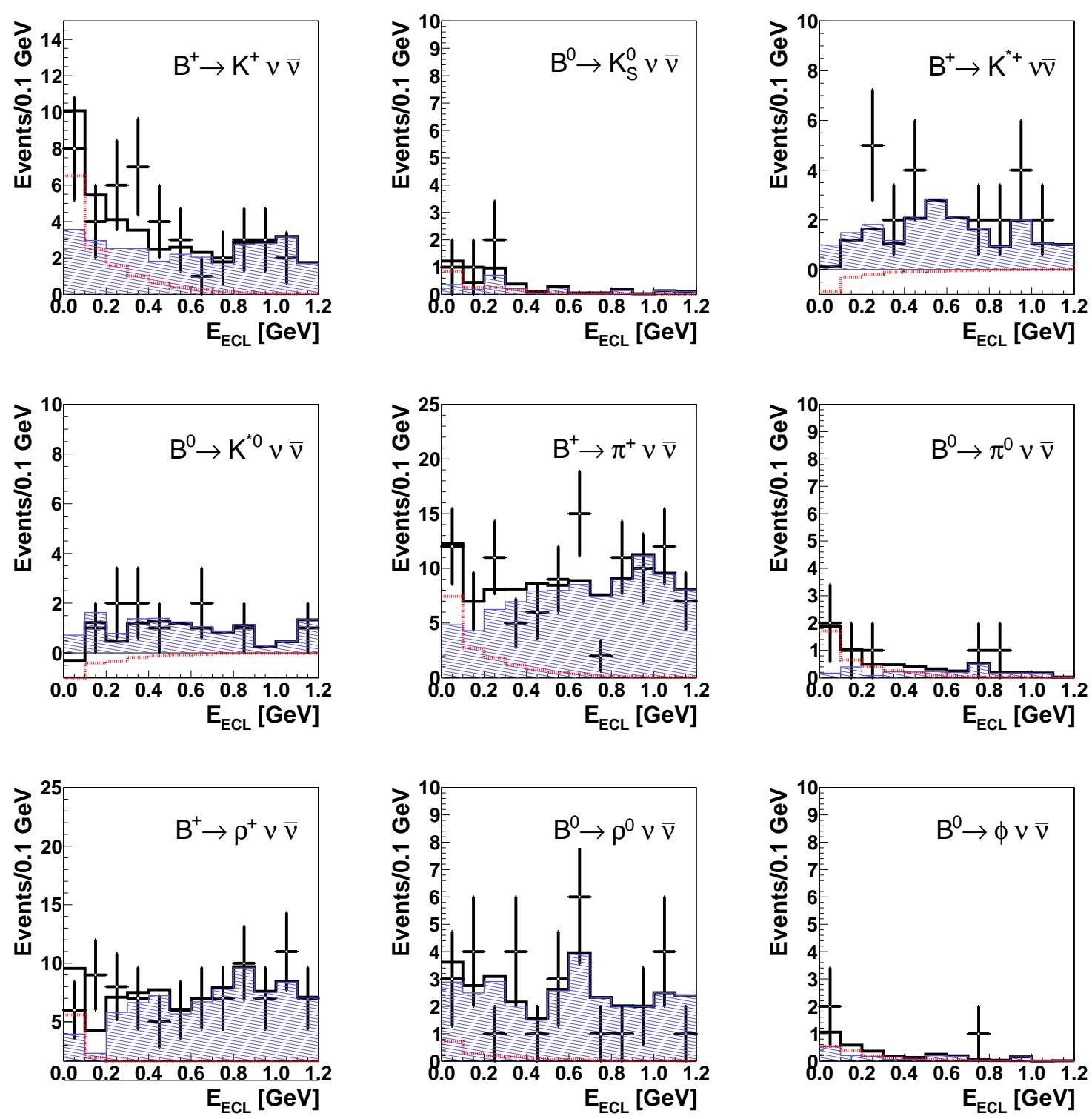

Figure 4: The $E_{E C L}$ distributions for $B \rightarrow h^{(*)} v \bar{v}$ decays. Points with error bars are data and the black solid line is the total fit result. The blue cross-hatched region is the background component and the red dashed line shows the signal contribution.

many models beyond the SM, for example supersymmetry, $v$ MSM or Grand Unification Theories. It is experimentally challenging to observe such heavy leptons, as they do not participate in strong, weak or electromagnetic interactions. Their only way of interaction is the mixing with left-handed neutrinos.

A search for $B \rightarrow l v_{h}(X), v_{h} \rightarrow l \pi$ decays $(l=e, \mu)$ was performed, with $M\left(v_{h}\right)=M\left(l_{1} \pi\right)$. The Feynman graphs with production and decay of a heavy neutrino are shown in fig. 5. The analysis was separated in two mass ranges: 'small' masses $<2 \mathrm{GeV}$, where only the exclusive decays 

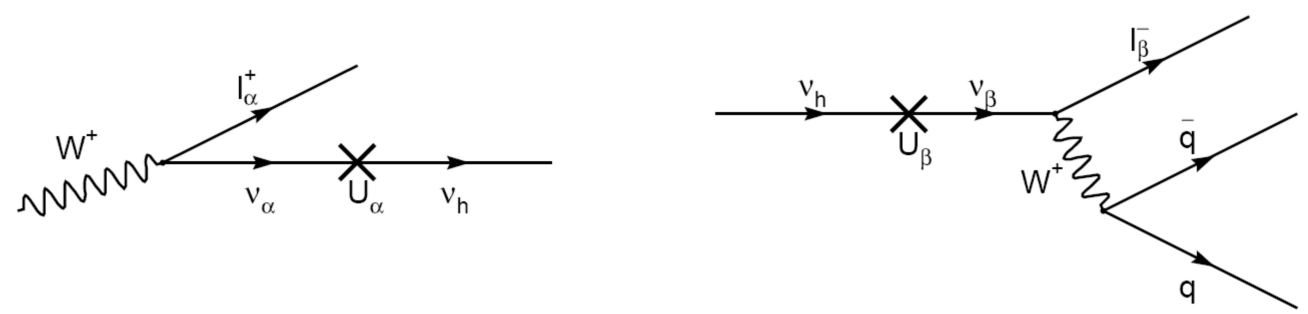

Figure 5: Heavy neutrino production and decay diagrams.

$B \rightarrow D^{(*)} l v_{h}$ was reconstructed, and 'large' masses $>2 \mathrm{GeV}$ with inclusive reconstruction. Strict selection criteria reduce the background by a factor $\sim 10^{6}$.
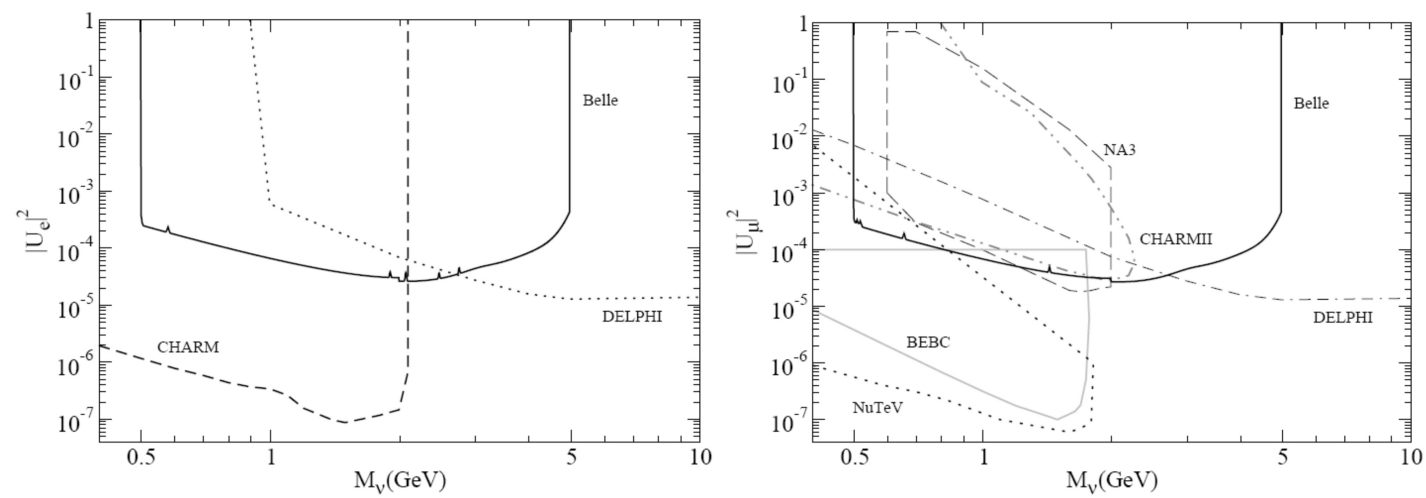

Figure 6: Comparison of obtained upper limits for with existing experimental results.

The observed events are well compatible with the background expectations, no significant signal was observed. An upper limit on mixing in the mass range $0.5-5 \mathrm{GeV} / \mathrm{c}^{2}$ was set and is compared to results of other experiments in fig. 6. Also an upper limit for product branching fraction was set: $\mathscr{B}\left(B \rightarrow l v_{h}(X)\right) \times \mathscr{B}\left(v_{h} \rightarrow l \pi\right)<6.6 \times 10^{-7}$ for $l=e, \mu$.

\section{References}

[1] G. Buchalla,A.J. Buras,Nuc. Phys. B 400, 225 (1993).

[2] A. Dedes,H. Dreiner,P. Richardson, Phys.Rev. D 65, 015001 (2002).

[3] G. Buchalla, Nuc. Phys. B 209, 137-142, (2010).

[4] M. Kreps, T. Kuhr, S. Neubauer, D. Zander, A. Zupanc, M. Feindt, F. Keller. NIM A 654, 432-440 (2011).

[5] K. -F. Chen et al, Phys. Rev. Lett. 99.22 (2007). 